\title{
Adsorption of $17 \alpha$-Ethinyl Estradiol and Bisphenol A to Graphene- Based Materials: Effects of Configuration of Adsorbates and the Presence of Cationic Surfactant
}

\author{
Fang Wang $\mathbb{D}^{1},{ }^{1}$ Wei Sha $\mathbb{D}^{1},{ }^{1}$ Xin Wang $\mathbb{D}^{1},{ }^{1}$ Yuntao Shang $\mathbb{D}^{1},{ }^{1}$ Lei Hou $\mathbb{D}^{2},{ }^{2}$ and Yao Li ${ }^{3}$ \\ ${ }^{1}$ Tianjin Key Laboratory of Water Resources and Environment, Tianjin Normal University, Tianjin 300387, China \\ ${ }^{2}$ College of Ecology and Environment, Southwest Forestry University, Kunming 650224, China \\ ${ }^{3}$ College of Environmental Science and Engineering, Ministry of Education Key Laboratory of Pollution Processes and \\ Environmental Criteria, Tianjin Key Laboratory of Environmental Remediation and Pollution Control, Nankai University, \\ Tianjin 300350, China
}

Correspondence should be addressed to Yao Li; hkliyao@nankai.edu.cn

Received 10 March 2021; Revised 1 April 2021; Accepted 7 April 2021; Published 21 April 2021

Academic Editor: Xue Tao Guo

Copyright (C) 2021 Fang Wang et al. This is an open access article distributed under the Creative Commons Attribution License, which permits unrestricted use, distribution, and reproduction in any medium, provided the original work is properly cited.

Endocrine-disrupting chemicals (EDCs) have attracted much attention in recent years. Graphene-based materials (GMs) have been deemed as excellent adsorbents for the removal of EDCs. The objective of the present study was to understand how the cationic surfactants (CTAB; cetyltrimethylammonium nitrate) affect the adsorption of EDCs (17 $\alpha$-ethinyl estradiol (EE2) and bisphenol A (BPA)) on graphene oxide (GO), reduced graphene oxides (RGOs), and the few-layered commercial graphene (CG). It was observed that the presence of CTAB showed different effects on the adsorption of EDCs to different GMs. The adsorption of EDCs on GO was enhanced because of the enhanced hydrophobicity of GMs after the adsorption of CTAB and the newly formed hemimicelles by the adsorbed CTAB, which could serve as the partition phase for EDCs. Moreover, the electron donoracceptor interaction and cation bridging effect of the $-\mathrm{NH}_{4}{ }^{+}$group of the adsorbed CTAB between EDCs and GMs could also enhance the adsorption of EDCs to GMs. With the increase of the extent of GM reduction, the adsorption enhancement by the presence of $\mathrm{CTAB}$ weakened. This could be attributed to the competition and pore blockage effect caused by the adsorbed CTAB. It is worth noting that the enhancement of CTAB on the adsorption of BPA to GMs was more profound than that of EE2. This is likely because the pore blockage effect plays a less important role in the adsorption of BPA due to its smaller molecular diameter and deformable structure.

\section{Introduction}

Endocrine-disrupting chemicals (EDCs), which are a class of the emerging contaminants, have been frequently detected in the aquatic environment in recent years [1-5]. They are capable of disturbing the physiological hormone systems of aquatic animals and human beings and increasing the risk of cancer, even at trace levels once ingested, and finally threaten the balance of the ecosystem [6, 7]. Thus, their transport and fate in the environment have received much attention.

Graphene-based materials (GMs), such as graphene oxide (GO), reduced graphene oxide (RGO), and graphene, have been applied in many fields due to their unique properties and have become an important member of the carbon nanomaterial family in recent years [8-12]. They have been proven to be excellent adsorbents for EDCs due to their large surface area, high hydrophobicity, and abundant functional groups [13-20]. For example, Jiang et al. [14] reported that $17 \beta$-estradiol showed strong adsorption affinities to GO, and the adsorption capacity of GO could reach $149.4 \mathrm{mgg}^{-1}$, which was much higher than that of single-walled carbon nanotubes and multiwalled carbon nanotubes. Jin et al. [15] reported that the reduction of GO could enhance its adsorption affinity towards 4-n-nonylphenol and BPA, which was 
attributed to the stronger hydrophobicity and $\pi$ electron density of RGO.

The adsorption affinities of EDCs to GMs could be affected by solution chemistry in the real aquatic environment. It was reported that cationic surfactants have been widely used in industrial manufactures and personal care products [21-26], and the concentration in the aquatic environment, especially in waste water, could reach $\mathrm{mg} \mathrm{L}^{-1}$ levels [22-24]. Thus, the coexisting cationic surfactant would affect the adsorption behavior of EDCs to GMs. The cationic surfactant could be adsorbed on the graphene surface via electronic attraction and hydrophobic interactions ([27]); thus, it could compete for the adsorption sites and decrease the adsorption of EDCs on GMs. With the concentration of the cationic surfactant increasing, it might form hemimicelles on the graphene surface and afford more adsorption sites [28]. This may enhance the adsorption of EDCs on GMs. Whether the cation surfactant would increase or decrease the adsorption of EDCs on GMs largely depends on the interaction between the cation surfactant and GMs, which is attributed to the properties of GMs [27]. For example, for the porous adsorbent materials, the cationic surfactant may block the access to the available pores inside and inhibit the adsorption of EDCs. Furthermore, as reported, the configuration of EDCs may also play a role in their adsorption to carbonaceous nanomaterials $[15,29]$. Thus, it is difficult to predict the actual effects of the cationic surfactant on the adsorption of EDCs to GMs. To the best of our knowledge, there are few studies on the cationic surfactant effects on the adsorption of organic contaminants to GMs. The only few researches focused on the surfactant effects were in regard to anionic surfactants [30]. As reported, the effects of the surfactant species on the adsorption of organic contaminants to carbonaceous nanomaterials were quite varied $[31,32]$. Therefore, in order to understand the complex interaction mechanisms among EDCs, GMs, and cation surfactants, relevant studies should be conducted.

The objective of this study was to examine the effects of the cationic surfactant on the adsorption affinities of GMs for typical EDCs. 17 $\alpha$-Ethinyl estradiol (EE2) and BPA were selected as the model EDCs because of their different hydrophobicity and steric configurations. Cetyltrimethylammonium nitrate (CTAB) was selected as the model cationic surfactant. Their adsorption behaviors on GO and two RGOs with different reduction extent were compared, and a commercial graphene (CG) was selected as a comparison because of its flat and few-layered structure. The mechanisms that controlled the effects of the cationic surfactant on EDC adsorption to GMs were systematically discussed. This study could provide a better understanding of the adsorption behavior of EDCs on GMs in the real aquatic system in the presence of cationic surfactants.

\section{Materials and Methods}

2.1. Materials. GO (>99\%) and CG (>99\%) was purchased from Plan Nano Materials Tech Co. (Tianjin, China). RGOs were obtained by reducing GO using the method that we reported before [32]. The reduction procedures were as fol- lows: First, $0.1 \mathrm{~g}$ GO powder was added in $400 \mathrm{~mL}$ deionized water and sonicated for $10 \mathrm{~min}$, to obtain a $\mathrm{GO}$ suspension. Then, a certain amount of L-ascorbic acid was added into the GO suspension and stirred in the dark for $72 \mathrm{~h}$ at $25 \pm$ $1^{\circ} \mathrm{C}$. After that, the reduced GO suspension was filtered through $0.22 \mu \mathrm{m}$ membrane filters. Finally, the materials on the filters were rinsed by deionized water for three times and collected. They were frozen and then dried in a freeze dryer (Labconco 6L, USA) at $-84^{\circ} \mathrm{C}$ for 5 days. According to the mass ratio of $\mathrm{L}$-ascorbic acid to $\mathrm{GO}$, the obtained RGOs were referred to as RGO_1 (1:1) and RGO_2 (10:1).

EE2 (98\%) and BPA (99\%) were purchased from SigmaAldrich (Shanghai, China). The phycochemical properties of EE2 and BPA are listed in Table S1. The EDC stock solutions were prepared in methanol and stored at $-20^{\circ} \mathrm{C}$. L-Ascorbic acid (99.5\%) was obtained from the VICTOR Co. (Tianjin, China). CTAB was purchased from Sinopharm Chemical Reagent Co., Ltd. (Shanghai, China). The critical micelle concentration and molecular structure of CTAB are summarized in Table S2. The inorganic salts $\left(\mathrm{NaH}_{2} \mathrm{PO}_{4} \cdot 2 \mathrm{H}_{2} \mathrm{O}\right.$ and $\left.\mathrm{Na}_{2} \mathrm{HPO}_{4} \cdot 12 \mathrm{H}_{2} \mathrm{O}\right)$ were obtained from Guangfu Technology Development Co. Ltd. (Tianjin, China). Glass optical fibers coated with polyacrylate (thickness $35 \mu \mathrm{m}$; volume $15.4 \mu \mathrm{L} \mathrm{m}^{-1}$ ) were purchased from Polymicro Technologies (Phoenix, AZ, USA).

2.2. Characterization of GO, RGOs, and CG. The morphological properties of GMs were examined with a transmission electron microscope (TEM) (JEM-2100, JEOL, Japan) and scanning electron microscopy (SEM) (S-3400N II, Hitachi, Japan). Surface elemental compositions of GMs were determined with X-ray photoelectron spectroscopy (XPS) (MultiLab 2000, Thermo Electron Corp., UK). The functional groups of GMs were analyzed by Fourier transform infrared transmission (FTIR) spectra (Bruker TENSOR 27 apparatus, Bruker Optics Inc., Germany). The graphitization degrees of GMs were evaluated by Raman spectra (RM2000, Renishaw Corp., UK). The $\zeta$ potential of GMs in the absence and presence of CTAB was measured by ZetaPALS (Brookhaven Instruments, USA) in a $10 \mathrm{mM} \mathrm{Na} \mathrm{HPO}_{4} / \mathrm{NaH}_{2} \mathrm{PO}_{4}$ buffer. The surface area and pore volume of GMs were determined from the surface area and porosimetry system (Micromeritics ASAP 2010, Micromeritics Co., USA). The relative hydrophobicity indexes of GMs were determined by the method reported by Walker et al. [33].

2.3. Adsorption Experiments. Adsorption experiments were conducted as follows [34]: First, $20 \mathrm{mg}$ of GMs was added in a $10 \mathrm{mM}$ phosphate buffer $\left(\mathrm{NaH}_{2} \mathrm{PO}_{4} / \mathrm{Na}_{2} \mathrm{HPO}_{4}\right.$, $\mathrm{pH}$ 5.96) to obtain a $50 \mathrm{mg} \mathrm{L}^{-1}$ suspension of GMs. Then, the GM suspension was added to a $20 \mathrm{~mL}$ glass vial. In the experiments that examined the effect of CTAB, an aqueous stock solution of CTAB was added to reach the concentration of $40 \mathrm{mg} \mathrm{L}^{-1}$. After that, the methanol stock solution of EE2 or BPA was added into the GM suspension. The volume percentage of the methanol stock solution was kept below $0.1 \%$ $(v / v)$ to minimize the cosolvent effects. The vials were equilibrated by tumbling at $8 \mathrm{rpm}$ in the dark for $14 \mathrm{~d}$ according to the preliminary experiment. To test the effects of surfactant 
concentrations, the concentrations were set as $5 \%, 10 \%, 20 \%$, $30 \%$, and $50 \%$ of the critical micelle concentration (CMC) of CTAB. The initial concentrations of EE2 and BPA were both set as $2.8 \mathrm{mg} \mathrm{L}^{-1}$. The aqueous concentrations of EE2 and BPA were measured by negligible depletion-solid-phase microextraction. The detailed procedures and the fiber sorption isotherms are shown in the Supplemental Data and Figure S1. All the adsorption isotherm experiments were run in duplicate, and the effects of surfactant concentration experiments were run in triplicate.

2.4. Analytical Methods. The concentrations of EE2 and BPA were determined using an Agilent 1200 high performance liquid chromatography system equipped with a symmetry reversed-phase $\mathrm{C}_{18}$ column $(4.6 \times 150 \mathrm{~mm}, 5 \mu \mathrm{m})$. EE2 and $\mathrm{BPA}$ were detected with a fluorescence detector. The excitation/emission wavelength for EE2 and BPA was $206 \mathrm{~nm} / 310 \mathrm{~nm}$ and $220 \mathrm{~nm} / 350 \mathrm{~nm}$, respectively. The mobile phase for both EE2 and BPA was $70 \%$ methanol and $30 \%$ deionized water with $10 \%$ acetic acid. The control experiment results showed that no peaks of potential degradation or transformation products of EE2 and BPA were detected, and the loss of EDCs due to adsorption on vials or other process was negligible. All data were analyzed and visualized using GraphPad Prism 6. Duncan's multiple range test was used to determine the difference significance (SPSS software, version 19.0, IBM Co., USA).

\section{Results and Discussion}

3.1. Characteristics of GO, RGOs, and CG. The selected physicochemical properties of GMs are summarized in Table 1, and the information of the surface element and functional group of GMs is shown in Figure 1. In general, surface $\mathrm{O}$ content of GMs was decreased by reduction, from $29.76 \%$ of GO to $26.71 \%$ of RGO_1 and $21.07 \%$ of RGO_2, while that of CG was $16.12 \%$, which was the lowest (Table 1 ). The $\mathrm{C}$ weight percentages of epoxy/alkoxy $(\mathrm{C}-\mathrm{O} / \mathrm{C}-\mathrm{O}-\mathrm{C})$ and carbonyl $(\mathrm{C}=\mathrm{O})$ of $\mathrm{GMs}$ were also decreased with the increase of the extent of reduction (Figures $1(\mathrm{a})-1(\mathrm{~d})$ ). The content of the O-functional groups on RGOs was higher than that of CG, which indicated that the reduction by L-ascorbic acid was not complete. It is noted that the $\mathrm{C}$ weight percentage of carboxyl $(\mathrm{O}-\mathrm{C}=\mathrm{O})$ was increased from $2.13 \%$ of $\mathrm{GO}$ to $4.69 \%$ of RGO_1 and 5.50\% of RGO_2. This is likely attributed to the adsorption of the reduction agent on RGOs as we reported before [32]. As shown in Figure 1(e), the peaks at 3422, 1735,1227 , and $1055 \mathrm{~cm}^{-1}$, which represented hydroxyl (O$\mathrm{H})$, carbonyl $(\mathrm{C}=\mathrm{O})$, and epoxy groups $(\mathrm{C}-\mathrm{O}-\mathrm{C})$ and alkoxy $(\mathrm{C}-\mathrm{OH})$, respectively, were significantly weakened with the increase of the extent of reduction $[35,36]$. This indicated that L-ascorbic acid could significantly decrease the amount of the polar functional groups on GO. The values of $\zeta$ potential of all GM solutions were negative, which was in the following order: GO < RGO_1 < RGO_2 < CG (Table 1). This is likely due to the decreased content of $\mathrm{O}$-functional groups as discussed before. The relative hydrophobicity indexes of the four GMs also increased with the increase of the extent of reduction. This was consistent with the above results.
The Raman spectra could reflect the graphitization degree of GMs $[37,38]$. As shown in Figure 1(f), the D and $\mathrm{G}$ bands of GO, RGO_1, RGO_2, and CG were all at 1340 and $1600 \mathrm{~cm}^{-1}$, and the intensity ratios of $D$ peak to $G$ peak $\left(I_{\mathrm{D}} / I_{\mathrm{G}}\right)$ increased slightly with the increase of the extent of reduction. This is likely because the reduction of GO could form new graphitic domains and increase surface heterogeneous atoms [39].

The morphological structures of GMs were examined by SEM and TEM. As shown in Figures 2(a) and 2(b), the surfaces of GO and CG were relatively flat and smooth, and their structures tended to be two-dimensional. With the increase of the extent of reduction, the graphene sheets of RGOs became wrinkled, fragmented, stacked, and porous, which were more like three-dimensional structures (Figures 2(e)$2(\mathrm{~h})$ ). This is likely because the L-ascorbic acid reduction could tear up the graphene layers to small fragments [32]. Meanwhile, the newly formed graphitic domains could enhance the $\pi$ electron density and hydrophobicity of GMs and finally enhance the $\pi-\pi$ interaction and hydrophobic interaction between the graphene layers and/or fragments. This could make them stacked together and form porous structures [32]. It is noted that the surface area and the pore volume of RGO_2 was much higher than those of GO and RGO_1, and the average pore diameter of RGO_2 was significantly decreased (Table 1). This is likely attributed to the smaller pores formed by reduction which could increase the inside surface of GMs.

3.2. Adsorption of EE2 and BPA to GMs. The adsorption isotherms of EE2 and BPA to GMs are shown in Figures 3(a) and 3(b), and the adsorption data were fitted with the Freundlich and Langmuir models (for the model details, see in the SI). The Freundlich model generally assumes that the adsorption is multilayered and heterogeneous, and the Langmuir model generally assumes that the adsorption is monolayered and the adsorption sites could reach saturation [40, 41]. The fitted parameters and the ranges of distribution coefficients $\left(K_{\mathrm{d}}\right)$ are summarized in Table 2 . It is noted that both models could fit the adsorption data well. The $n$ values of all the GMs in the Freundlich model were less than 1, which could be due to the heterogeneous adsorption sites on GMs.

As shown in Figure 3 and Table 2, RGOs exhibited stronger adsorption affinities than GO. The adsorption affinities $\left(K_{\mathrm{d}}\right.$ values in Table 2$)$ as well as the adsorption capacities of GMs $\left(Q_{\max }\right.$ values in Table 2$)$ were in the following order: RGO_2 > RGO_1 > GO. To better understand the adsorption mechanism excluding the effect of the amount of the adsorption sites, the adsorption isotherms were normalized by the BET surface area of GMs (Figures 3(c) and 3(d)). After the normalization, the adsorption affinities of EDCs on RGOs were still higher than those on GO. This may be attributed to two reasons: (1) the hydrophobicity of GMs reflected by the relative hydrophobicity index (Table 1) increased with the increase of reduction extent of GMs, and the hydrophobic interaction between GMs and EDCs was strengthened; (2) the density of $\pi$ electrons reflected by the value of $I_{\mathrm{D}} / I_{\mathrm{G}}$ (Table 1) of GMs also increased with the increase of the reduction extent of GMs; thus, the $\pi-\pi$ electron donor- 
TABLE 1: Selected physicochemical properties of GO, RGO_1, RGO_2, and CG.

\begin{tabular}{|c|c|c|c|c|c|c|c|c|c|}
\hline \multirow{2}{*}{$\begin{array}{l}\text { Graphene } \\
\text { materials }\end{array}$} & \multicolumn{3}{|c|}{$\begin{array}{c}\text { Element } \\
\text { composition } \\
(\mathrm{wt} \%)^{\mathrm{a}}\end{array}$} & \multirow{2}{*}{$\begin{array}{c}\text { Relative } \\
\text { hydrophobicity } \\
\text { index }\end{array}$} & \multirow[t]{2}{*}{$\zeta$ potential $^{\mathrm{c}}$} & \multicolumn{2}{|c|}{ Pore volume $\left(\mathrm{cm}^{3} \mathrm{~g}^{-1}\right)^{\mathrm{d}}$} & \multirow{2}{*}{$\begin{array}{c}\text { Average pore } \\
\text { diameter }(\mathrm{nm})^{\mathrm{d}}\end{array}$} & \multirow{2}{*}{$\begin{array}{l}\text { BET surface area } \\
\qquad\left(\mathrm{m}^{2} \mathrm{~g}^{-1}\right)^{\mathrm{d}}\end{array}$} \\
\hline & $\mathrm{C}$ & $\mathrm{O}$ & $\mathrm{C} / \mathrm{O}$ & & & Micropore & $\begin{array}{l}\text { Mesopore } \\
\text { macropore }\end{array}$ & & \\
\hline GO & 69.38 & 29.76 & 2.33 & $0.05 \pm 0.01$ & $-34.40 \pm 3.73$ & 0.004 & 0.050 & 13.73 & 68.56 \\
\hline RGO_1 & 70.38 & 26.71 & 2.63 & $0.20 \pm 0.03$ & $-31.70 \pm 0.20$ & 0.015 & 0.030 & 6.07 & 56.70 \\
\hline RGO_2 & 78.04 & 21.07 & 3.70 & $0.32 \pm 0.04$ & $-24.13 \pm 0.86$ & 0.038 & 0.393 & 3.75 & 102.24 \\
\hline CG & 79.39 & 16.12 & 4.92 & $0.93 \pm 0.10$ & $-23.81 \pm 0.91$ & 0.018 & 0.178 & 10.78 & 78.51 \\
\hline
\end{tabular}

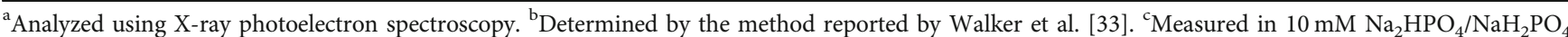
buffer. ${ }^{\mathrm{d}}$ Analyzed using surface area and porosimetry system.

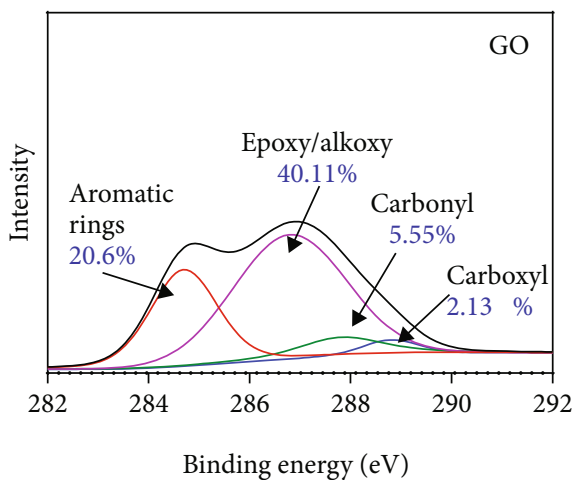

(a)

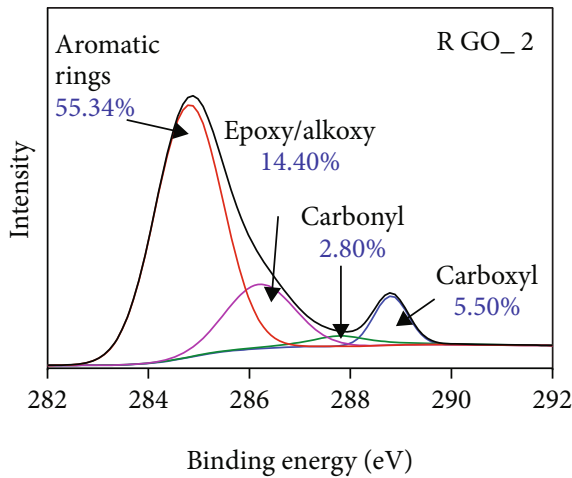

(c)

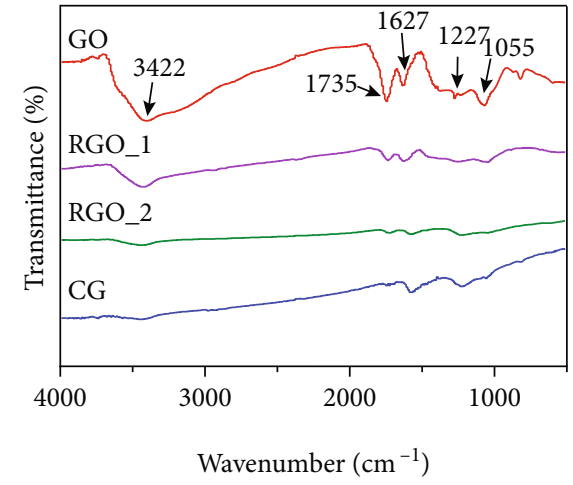

(e)

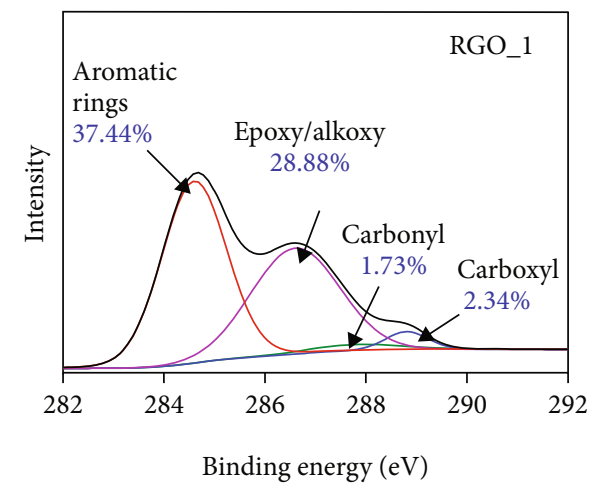

(b)

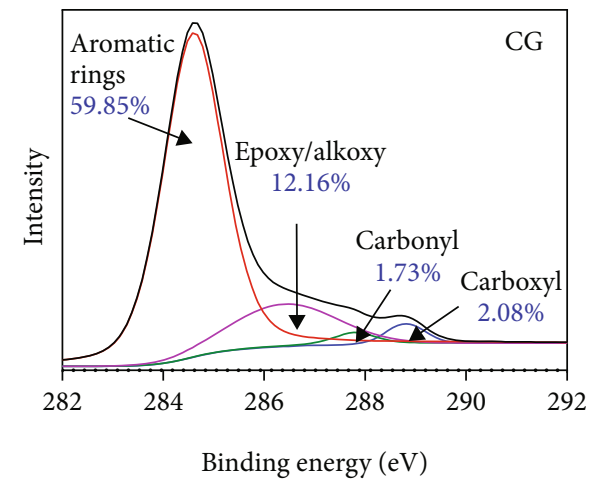

(d)

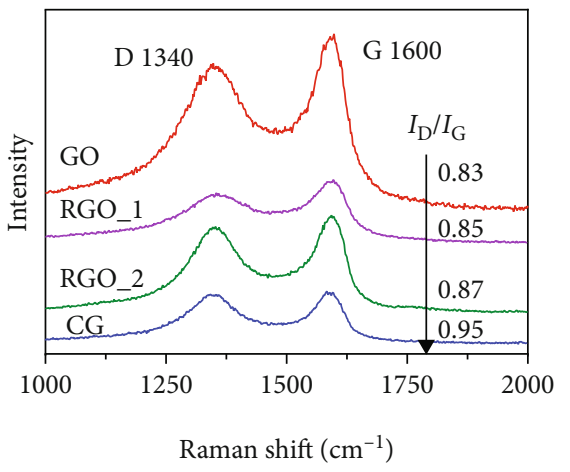

(f)

FIGURE 1: X-ray photoelectron spectroscopy (XPS) curve fit of C1s spectra of GMs (a-d); Fourier transform infrared spectra (FTIR) of GMs (e); Raman spectra of GMs (f). 


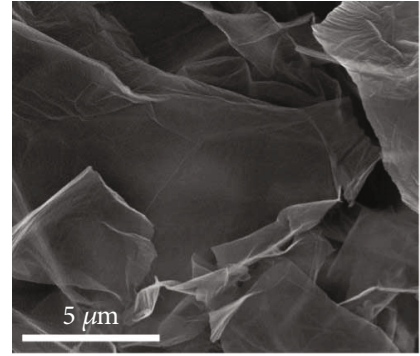

(a)

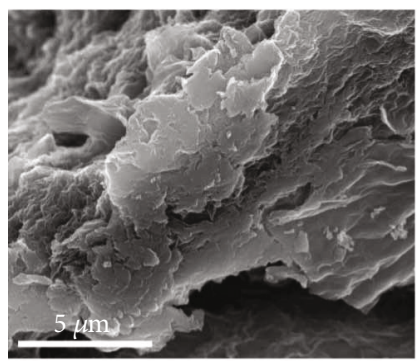

(c)

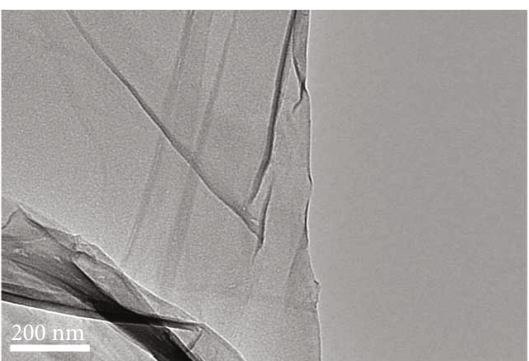

(e)

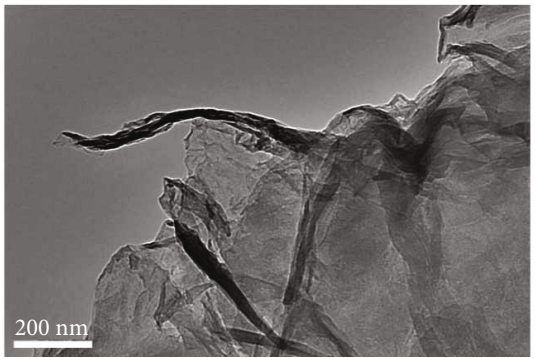

(g)

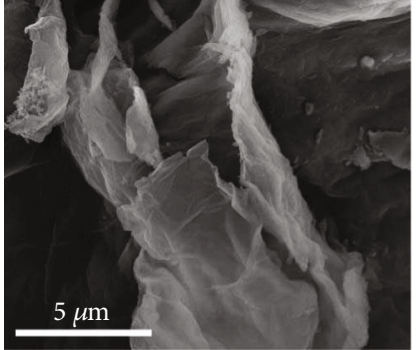

(b)

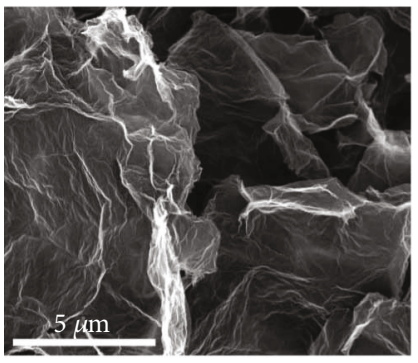

(d)

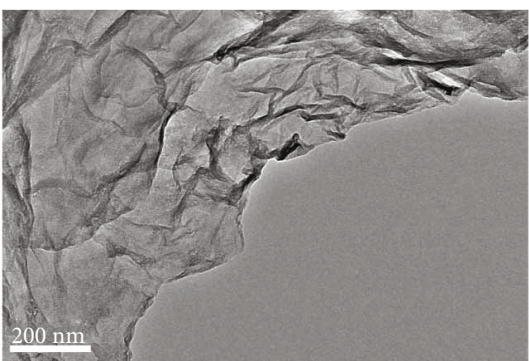

(f)

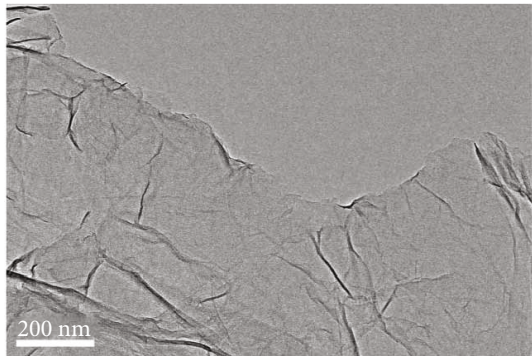

(h)

FIGURE 2: Scanning electron microscopy (SEM) (a-d) and transmission electron microscopy (TEM) (e-h) images of GO (a, e), RGO_1 (b, f), RGO_2 (c, g), and CG (d, h).

acceptor (EDA) interaction between GMs and EDCs was enhanced. Hydrophobic and $\pi-\pi$ EDA interactions were reported as the dominant effect between RGO and EDCs.

To further compare the respective contribution of hydrophobic interaction with $\pi-\pi$ EDA interaction, the normalized adsorption isotherms of EDCs to GMs were further normalized by relative hydrophobicity indexes and $I_{\mathrm{D}} / I_{\mathrm{G}}$ values of GMs, respectively (Figure S2). It is noted that the adsorption isotherms normalized by relative hydrophobicity indexes were significantly closely shifted, and the shift of the adsorption isotherms that was normalized by $I_{\mathrm{D}} / I_{\mathrm{G}}$ was very slight, and it could even be negligible for EE2 (Figure S2d).
This proved that the hydrophobic interaction is more important than the $\pi-\pi$ EDA interaction in the adsorption process of EDCs to GMs.

It is noted that although the hydrophobicity and the $\pi$ electron density of CG were the highest (Table 1), the adsorption capacities as well as the adsorption affinity of EE2 on CG were still lower than those on RGO_2, especially at high equilibrium concentrations. This could be attributed to the different structures of CG and RGO_2. The adsorption sites of CG were exposed because of the open-layered structure; thus, the EE2 molecules could be easily adsorbed at the low concentrations. With the increase of the EE2 concentration, the 


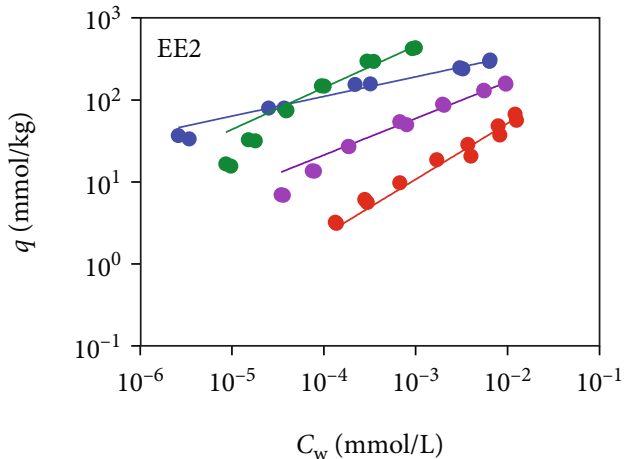

(a)

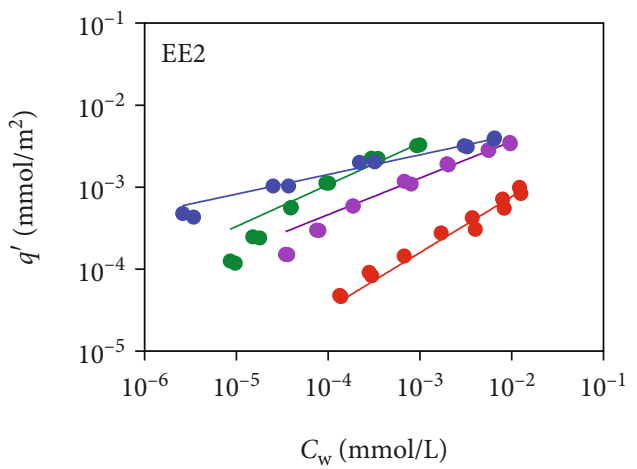

(c)

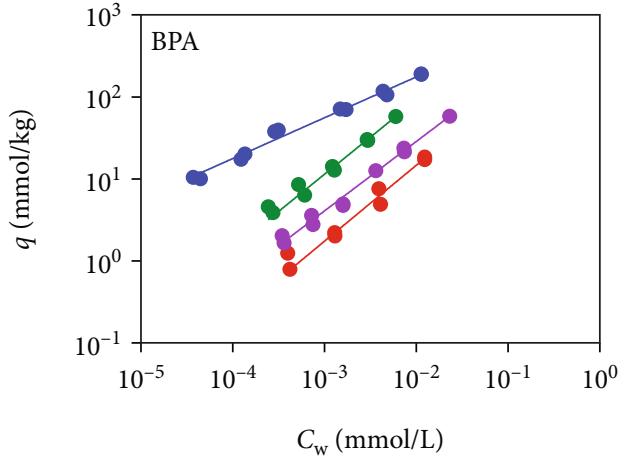

(b)

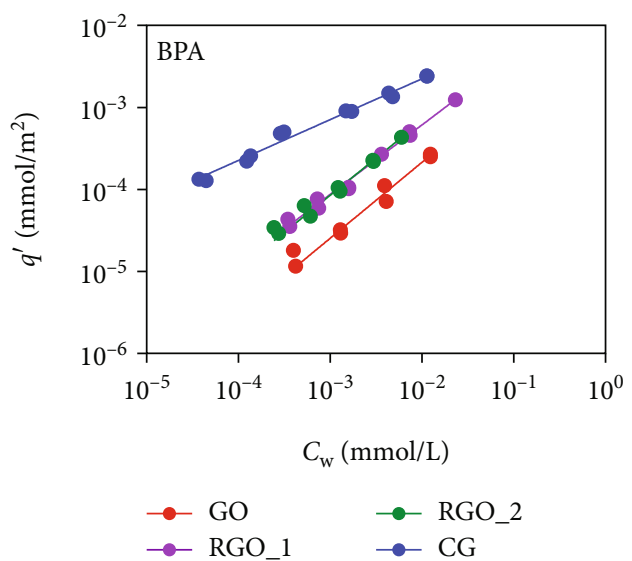

(d)

FIgURE 3: Adsorption isotherms of EE2 and BPA to GMs (a, b) and those normalized by BET surface area (c, d).

TABLE 2: Summary of adsorption parameters [Freundlich model coefficients $\left(K_{\mathrm{F}}\right.$ and $\left.n\right)$ and distribution coefficients $\left(K_{\mathrm{d}}\right)$ ] obtained from adsorption results.

\begin{tabular}{|c|c|c|c|c|c|c|c|c|c|}
\hline \multirow{2}{*}{ Adsorbate } & \multirow{2}{*}{ Adsorbent } & \multirow{2}{*}{ Background } & \multicolumn{3}{|c|}{ Freundlich model } & \multicolumn{3}{|c|}{ Langmuir model } & \multirow{2}{*}{$\log K_{\mathrm{d}}\left(\mathrm{L} \mathrm{kg}^{-1}\right)$} \\
\hline & & & $K_{\mathrm{F}}\left(\mathrm{mmol}^{1-n} \mathrm{~L}^{n} \mathrm{~kg}^{-1}\right)^{\mathrm{a}}$ & $n^{\mathrm{a}}$ & $R^{2}$ & $Q_{\max }\left(\mathrm{mmol} \mathrm{kg}^{-1}\right)^{\mathrm{a}}$ & $K_{\mathrm{L}}^{\mathrm{a}}$ & $R^{2}$ & \\
\hline \multirow{8}{*}{ EE2 } & \multirow{2}{*}{ GO } & Buffer & $1200 \pm 450$ & $0.68 \pm 0.08$ & 0.958 & $140 \pm 51$ & $61 \pm 35$ & 0.944 & $3.65-4.38$ \\
\hline & & CTAB & $12000 \pm 1100$ & $0.65 \pm 0.01$ & 0.998 & $590 \pm 28$ & $310 \pm 29$ & 0.997 & $4.86-5.42$ \\
\hline & \multirow{2}{*}{ RGO_1 } & Buffer & $1300 \pm 120$ & $0.45 \pm 0.02$ & 0.992 & $180 \pm 8.6$ & $510 \pm 74$ & 0.984 & $4.21-5.31$ \\
\hline & & CTAB & $11000 \pm 970$ & $0.65 \pm 0.02$ & 0.997 & $560 \pm 29$ & $300 \pm 28$ & 0.997 & $4.79-5.43$ \\
\hline & \multirow{2}{*}{ RGO_2 } & Buffer & $15000 \pm 4300$ & $0.51 \pm 0.04$ & 0.987 & $540 \pm 10$ & $3800 \pm 200$ & 0.998 & $5.64-6.33$ \\
\hline & & СТAB & $15000 \pm 6300$ & $0.74 \pm 0.08$ & 0.960 & $730 \pm 220$ & $130 \pm 61$ & 0.961 & $4.66-5.23$ \\
\hline & \multirow{2}{*}{ CG } & Buffer & $1000 \pm 86$ & $0.24 \pm 0.01$ & 0.973 & $280 \pm 18$ & $6700 \pm 2400$ & 0.918 & $4.67-7.15$ \\
\hline & & CTAB & $2300 \pm 170$ & $0.44 \pm 0.01$ & 0.996 & $320 \pm 28$ & $520 \pm 140$ & 0.960 & $4.54-6.44$ \\
\hline \multirow{8}{*}{ BPA } & \multirow{2}{*}{ GO } & Buffer & $1000 \pm 380$ & $0.93 \pm 0.08$ & 0.985 & $150 \pm 17$ & $11 \pm 1.5$ & 0.984 & $3.08-3.49$ \\
\hline & & CTAB & $12000 \pm 930$ & $0.74 \pm 0.01$ & 0.998 & $730 \pm 54$ & $96 \pm 11$ & 0.997 & $4.83-5.52$ \\
\hline & \multirow{2}{*}{ RGO_1 } & Buffer & $1400 \pm 130$ & $0.85 \pm 0.02$ & 0.997 & $210 \pm 26$ & $17 \pm 2.8$ & 0.998 & $3.27-3.77$ \\
\hline & & CTAB & $9600 \pm 800$ & $0.73 \pm 0.03$ & 0.995 & $660 \pm 66$ & $95 \pm 15$ & 0.994 & $4.74-5.56$ \\
\hline & \multirow{2}{*}{ RGO_2 } & Buffer & $6000 \pm 1000$ & $0.91 \pm 0.03$ & 0.995 & $420 \pm 19$ & $27 \pm 1.4$ & 0.994 & $3.99-4.27$ \\
\hline & & CTAB & $14000 \pm 1300$ & $0.94 \pm 0.02$ & 0.998 & $2100 \pm 700$ & $9.7 \pm 3.5$ & 0.998 & $4.50-4.93$ \\
\hline & \multirow{2}{*}{ CG } & Buffer & $1700 \pm 210$ & $0.50 \pm 0.02$ & 0.991 & $260 \pm 34$ & $220 \pm 69$ & 0.950 & $4.18-5.45$ \\
\hline & & CTAB & $3500 \pm 170$ & $0.64 \pm 0.01$ & 0.999 & $380 \pm 37$ & $93 \pm 17$ & 0.989 & $4.46-5.47$ \\
\hline
\end{tabular}

a Values after " \pm ” sign indicate relative standard deviation. 
adsorption sites of CG became saturated. However, the wrinkled surface of RGO_2 could form new pores inside which could offer more adsorption sites. Thus, the EE2 molecules could be adsorbed on RGO_2 via the pore filling mechanism. Moreover, the content of O-functional groups on RGO_2 was higher than that on CG. The H-bonding interaction between EE2 and RGO_2 is stronger than that between EE2 and CG. The relatively stronger H-bonding on RGO_2 could also offset part of the hydrophobicity effect and $\pi-\pi$ EDA interaction effect and made the adsorption of EE2 on RGO_2 stronger.

Interestingly, the reduction of GO showed stronger influence on EE2 than on BPA. For example, when the EDC equilibrium concentration was at $10^{-3} \mathrm{mg} \mathrm{L}^{-1}$, the $K_{\mathrm{d}}$ value of EE2 on RGO_2 was increased by the factor of 94.1 compared with that on GO, whereas that of BPA was increased by the factor of 9.0. This is likely due to the higher hydrophobicity of EE2 compared to BPA $\left(\log K_{\mathrm{OW}-\mathrm{EE} 2}=4.15, \log K_{\mathrm{OW}-\mathrm{BPA}}=2.2\right)$, and the hydrophobic interactions have greater weight in the adsorption process of the more hydrophobic compound. Thus, the reduced hydrophobicity of GO showed more influence on EE2 adsorption. Notably, the $n$ value of EE2 adsorption isotherms was lower than that of BPA on each GM, and the reduction effect on the decrease of the $n$ values of EE2 was more obvious. For example, the $n$ values of EE2 adsorption isotherms were decreased by $34.6 \%$ and $25.4 \%$ for RGO_1 and RGO_2, respectively, whereas those of BPA adsorption isotherms were decreased by $7.8 \%$ and $6.1 \%$, respectively. This is likely because the critical molecular diameter and molecular volume of EE2 are bigger than those of BPA, and the two benzene rings of BPA could rotate and fit the surface of GMs, lowering the adsorption energy [15, 29], whereas the EE2 molecule does not have this advantage. With the increase of the extent of reduction, the pore width of GMs was smaller, and the surface of GMs became wrinkled. Thus, the EE2 molecule was limited to going inside the pore via molecular sieve, whereas the BPA molecule could still penetrate the micropores via the rotated structure. This also explained why the adsorption capacities $\left(Q_{\max }\right)$ of BPA on RGOs are higher than those of EE2 (Table 2).

3.3. Effects of CTAB on Adsorption of EDCs Were Varied on Different GMs. The adsorption isotherms of EDCs to GMs in the presence of CTAB are shown in Figure 4, and the corresponding fitting results of adsorption data to the Freundlich and Langmuir models are summarized in Table 2. In general, $\mathrm{CTAB}$ showed different effects on the adsorption of EDCs to different GMs. For example, CTAB enhanced the adsorption of EE2 on GO and RGO_1, whereas it inhibited that of EE2 on RGO_2 and CG. The $Q_{\max }$ of EE2 in the presence of CTAB was increased by the factor of 4.2, 3.1, 1.4, and 1.1 on GO, RGO_1, RGO_2, and CG, respectively. These results also indicated that the adsorption enhancement in the presence of $C T A B$ decreased with the increase of the extent of GM reduction. The adsorption of BPA in the presence of CTAB showed the similar trend.

According to the previous studies, cationic surfactants may alter the adsorption of organic contaminants via the following mechanisms: (1) increasing the solubility of organic contaminants, (2) competing organic contaminants with the adsorption sites, (3) changing the surface or aggregation properties of the adsorbent surface, (4) forming hemimicelles in which organic contaminants could partition in $[30,31,42$, 43]. In this experiment, the initial concentration of $\mathrm{CTAB}$ was selected as $40 \mathrm{mg} \mathrm{L}^{-1}$, which was much lower than its critical micelle concentration (Table S2). The effects of CTAB concentrations on the solubility of EDCs are shown in Figure S3. The result indicated that the selected concentration of CTAB had negligible effect on the solubility of EE2 and BPA, and the solubilization effect of $\mathrm{CTAB}$ on EE2 and BPA could be ignored. Thus, the different effects of CTAB on EDC adsorption on varied GMs could be attributed to the adsorption affinities of CTAB on GMs. Therefore, the adsorption affinities of $\mathrm{CTAB}$ on the four GMs were detected.

As shown in Figure 54 , the adsorption affinity of CTAB followed the order $\mathrm{GO}>\mathrm{RGO} \_1>\mathrm{CG}>\mathrm{RGO}$ 2. It has been reported that the cationic surfactant could interact with GMs via electrostatic attraction supplemented by hydrophobic interaction [27]. In this study, the positively charged $\mathrm{CTAB}$ interacted with the negatively charged surface of GMs via electrostatic attraction. With the reduction extent of GO, the amounts of O-functional groups and the negative charge of GMs decreased (Table 1), and the electrostatic attraction between GMs and $C T A B$ also decreased. Thus, the amounts of the adsorbed $\mathrm{CTAB}$ decreased with the increase of the GO reduction extent. It is noted that although the $\zeta$ potential of CG was less negative compared with that of RGO_2 (Table 1), the adsorption affinity of CTAB on CG was stronger than that on RGO_2. This is likely because the L-ascorbic acid reduction could result in the formation of the micropores and wrinkles on the surface of RGOs. The long carbon chain of CTAB may crisscross at the access of the micropores and finally block the micropores [32]. Thus, CTAB could not go into the available adsorption sites inside, and the adsorption on RGOs was inhibited. However, for CG, the open-layered structure made the adsorption sites exposed. Thus, CG could offer more adsorption sites for CTAB compared with RGOs. Furthermore, the relatively stronger hydrophobicity of CG could strengthen the hydrophobic interaction between the long carbon chain of CTAB and the graphene domain of CG [27]. This could also enhance the adsorption of CTAB on CG.

As shown in Figure 4(a), the adsorption of EDCs was enhanced by more than $1 \log$ unit in the presence of CTAB, although $C T A B$ occupied the highest amount of adsorption sites on GO. As shown in Table S3, after adsorbed CTAB, the $\zeta$ potential of GO became less negative. This indicated that the hydrophobicity of GO increased, and this could enhance the hydrophobic interaction between EDC and GO in the presence of CTAB. Meanwhile, it was reported that CTAB molecules were flatly adsorbed on the surface of GO at low surfactant concentration and competed with EDCs for the adsorption sites [27]. With the increase of surfactant concentration, the alkyl chains of CTAB molecules started to leave the surface of GO and form hemimicelles [27]. These newly formed hemimicelles could offer adsorption 


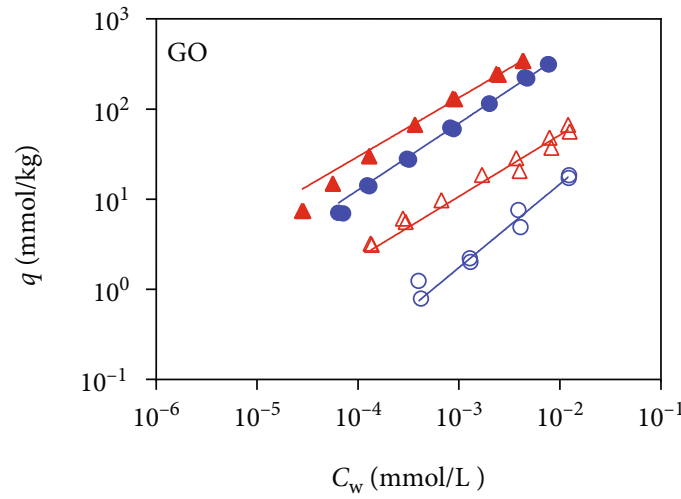

(a)

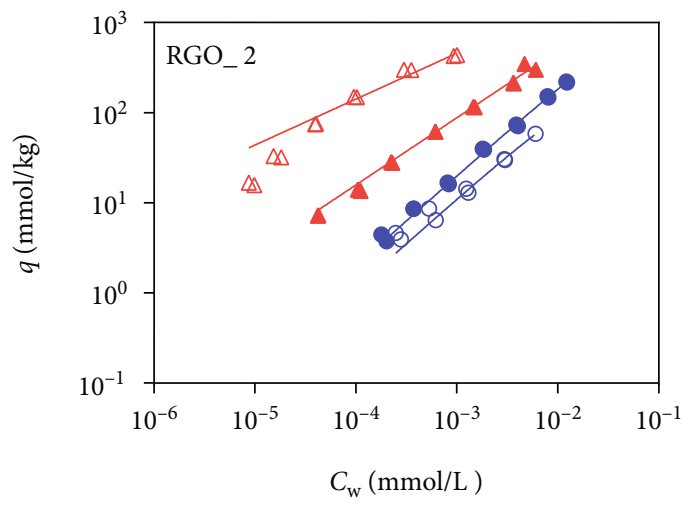

(c)

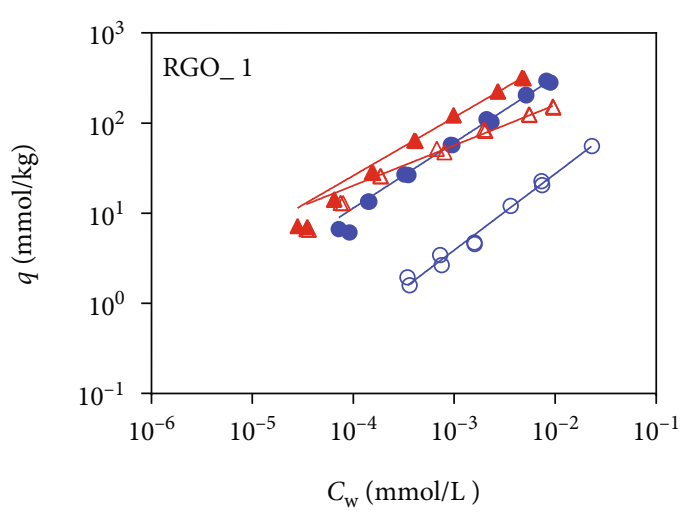

(b)

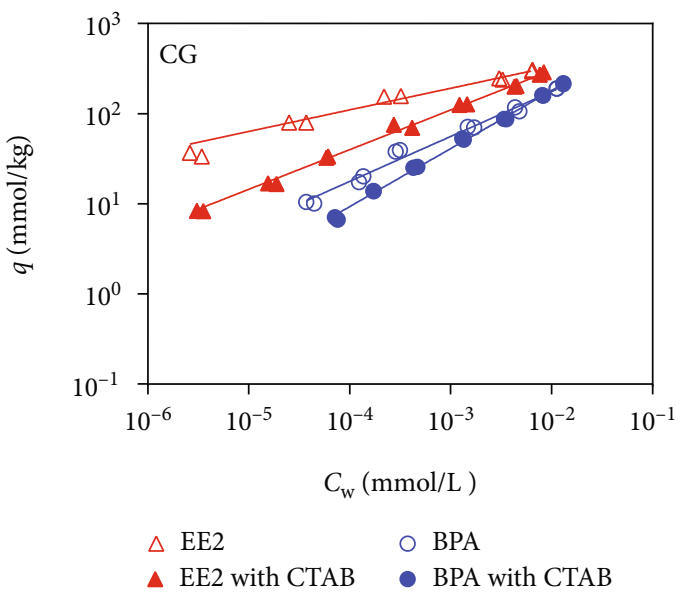

(d)

FIgURE 4: Adsorption isotherms of EE2 and BPA to GMs in the absence and presence of CTAB.

sites for organic contaminant partitioning [28]. Thus, in this study, it is supposed that when the amount of the adsorbed CTAB was $6.37 \times 10^{5} \mathrm{mg} \mathrm{kg}^{-1}$ on GO, the hemimicelles were formed on the GO surface and offered more available sites for EDCs to partition. Meantime, the $-\mathrm{NH}_{4}{ }^{+}$group of CTAB adsorbed on GMs could also act as a $\pi$ electron donor and enhance the $\pi-\pi$ EDA interaction between GMs and EDCs. On the other hand, the exposed $-\mathrm{NH}_{4}{ }^{+}$group of adsorbed $\mathrm{CTAB}$ could also interact with the $-\mathrm{OH}$ group of EDCs via Lewis acid-base interactions and act as a cation bridge agent between EDCs and the surface groups of GO. These also enhanced the EDC adsorption. It is noted that GO could aggregate in the presence of CTAB because of the opposite charge of $\mathrm{GO}$ and $\mathrm{CTAB}$, and with the increase of CTAB concentration, the aggregation is more profound. On the one hand, the aggregation of graphene sheets could decrease the adsorption site. On the other hand, the newly formed high-energy adsorption sites due to the aggregation could enhance the adsorption [44]. To further verify the mechanisms, the effects of different CTAB concentrations on EDC adsorption were analyzed (Figure 5). As shown in Figure 5(a), with the increase of the CTAB concentration, the enhancement effect by CTAB on GO was strengthened. This indicated that the competition effect and decreased adsorption site due to the aggregation of GO in the presence of CTAB play a minor role in the observed adsorption enhancement.

With the increase of the extent of GM reduction, the enhancement of CTAB to the adsorption of EDCs to GMs had been weakened (Figures 4(b) and 4(c)). As shown in Figure S4, the adsorbed amount of CTAB decreased to 3.84 $\times 10^{5}$ and $1.06 \times 10^{5} \mathrm{mg} \mathrm{kg}^{-1}$ on RGO_1 and RGO_2, respectively. The hemimicelles of $\mathrm{CTAB}$ formed on the graphene surface also decreased; thus, the adsorption enhancement by the hemimicelles was weakened, and the competition effect became the main inhibition mechanism on RGOs. Moreover, with the increase of the extent of GM reduction, the pore width became smaller, and the adsorbed CTAB crisscrossed at the access of the pore as mentioned before. This could also block the access of EDC molecules. This indicated that for the three-dimensional RGOs, the pore blockage effect is another main mechanism. In order to better illustrate the dominant mechanisms, the effects of CTAB concentrations on the adsorption of the two EDCs to graphene surfaces were summarized in Figure 6 . Interestingly, with the increase of CTAB concentration, the effect of CTAB on EE2 adsorption changed from inhibition effect to enhancement effect on RGO_1 (Figure 5(b)). This indicated that the mechanisms of EE2 adsorption in the presence of CTAB varied at different CTAB concentrations. 


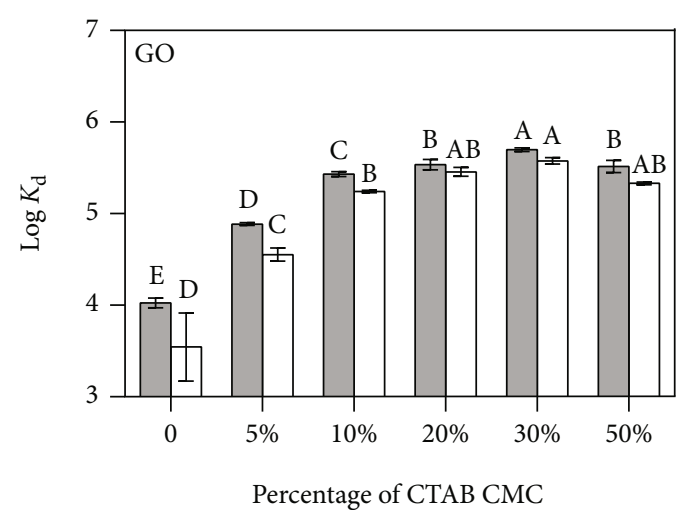

(a)

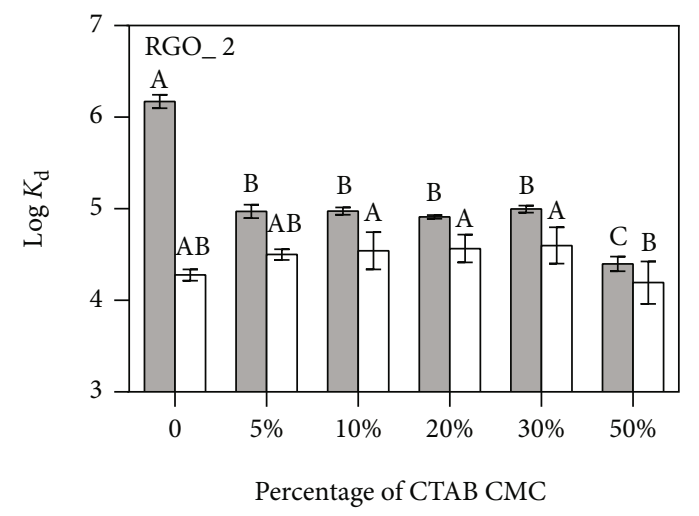

(c)

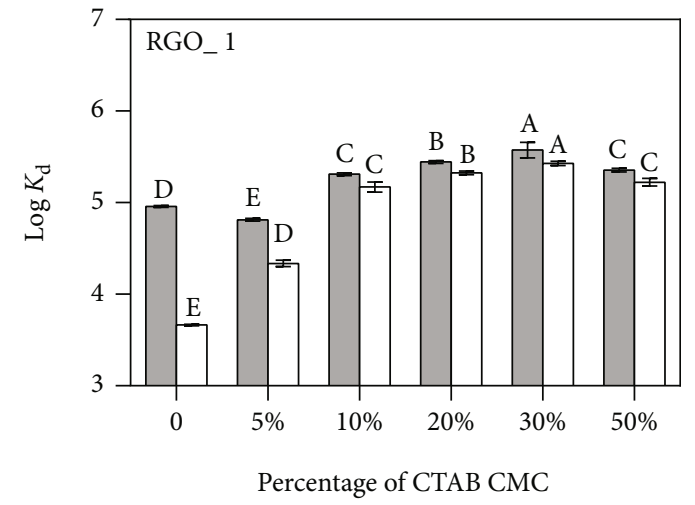

(b)

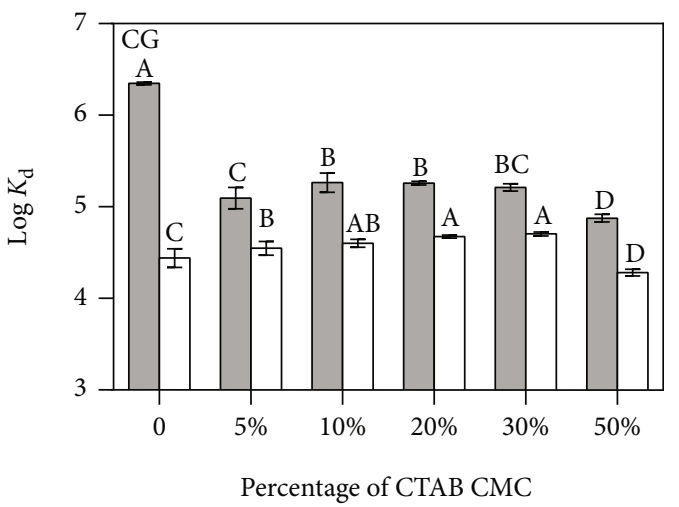

$\square$ EE2

(d)

FIGURE 5: Adsorption coefficients of EE2 and BPA to GMs under different concentrations of CTAB. Different letters marked on the top of the bars indicate significant differences in each group $(p<0.05)$.

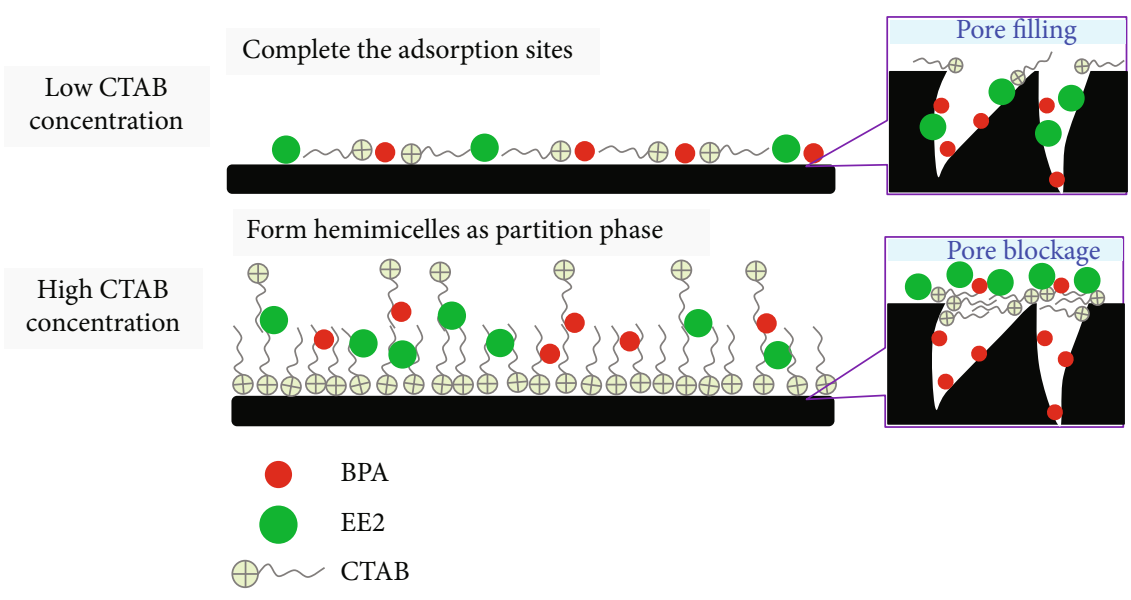

FIGURE 6: Schematic graph of mechanisms controlling the effects of CTAB concentration.

At a low CTAB concentration, the main mechanism was competition/pore blockage as mentioned before. With the increase of CTAB concentration, the hemimicelle effect became the main mechanism. When the concentration of $\mathrm{CTAB}$ reached $170 \mathrm{mg} \mathrm{L}^{-1}$, the adsorption affinities of EDCs on all GMs decreased. This is likely because of the solubilization effect (Figure S2).

Notably, the adsorption of CTAB on CG was stronger than that on RGO_2, and the $\zeta$ potential was similar Compete the adsorption sites with EDCs (Table S3); however, 
the inhibition effect of CTAB on the adsorption of EE2 to CG was weaker than that to RGO_2, especially at a high concentration of EE2. This may be attributed to the openlayered structure of CG. The inhibition caused by the pore blockage effect did not play a role for the two-dimensional CG.

\subsection{Effects of CTAB on Adsorption of BPA Were Different} from those on EE2. As shown in Figures 4 and 5, the enhancement effect of CTAB on BPA was stronger than that on EE2. For example, when the aqueous EDC concentration was $10^{-}$ ${ }^{3} \mathrm{mmol} \mathrm{L}^{-1}$, the value of $K_{\mathrm{d}}$ of BPA on GO increased by 43.2 times in the presence of CTAB, whereas that of EE2 increased by 12.5 times. With the increase of the extent of $\mathrm{GM}$ reduction, the enhancement effect of $\mathrm{CTAB}$ on BPA decreased. On RGO_2, it still had a bit of an enhancement effect, whereas it inhibited the adsorption of EE2 on RGO_ 2. This is likely because the structure of BPA has two benzene rings and two phenol groups, and the $\pi$ electron density is higher than that of EE2; therefore, the $\pi-\pi$ EDA interaction between GMs and BPA is stronger. Meanwhile, the Lewis acid-base interaction between $\mathrm{NH}_{4}{ }^{+}$of CTAB and BPA is stronger due to more phenol groups on $\mathrm{BPA}$, and the bridge effect between $\mathrm{CTAB}$ and BPA is stronger. Moreover, the molecular diameter of BPA is smaller than that of EE2, and the structure of BPA could change the angle of the structure of two benzene rings, in order to fit the surface of the adsorbate better [29]. Thus, the pore blockage effect on the adsorption of BPA was not obvious (Figure 6). These all lead to the stronger enhancement effect of CTAB on BPA adsorption.

\section{Conclusions}

The reduction of GO by L-ascorbic acid could increase the hydrophobicity and the amount of micropores of GO and further enhanced the adsorption affinities of GMs for both EE2 and BPA. The presence of the cationic surfactant, i.e., CTAB, showed different effects on the adsorption of EDCs to different GMs. It was the enhancement effect on GO, and with the increase of the reduction extent, the enhancement effect of CTAB was weakened and finally became the inhibition effect. This could be attributed to the combination effects of the following aspects: (1) CTAB was flatly adsorbed on the surface of GMs at a low surfactant concentration and competed with EDCs for the adsorption sites; (2) with the increase concentration of $\mathrm{CTAB}$, the hemimicelles formed on the surface of GMs could serve as a partition phase for EDCs; (3) the adsorbed CTAB on the surface of RGOs crisscrossed at the access of the pore and blocked the entrance of EDC molecules, thus inhibiting the adsorption of EDCs; and (4) the adsorbed CTAB could enhance the adsorption of EDCs to GMs by strengthening the hydrophobic effects, $\pi$ $\pi$ EDA interactions, and also cation bridging. Furthermore, the configuration of EDCs also played an important role. The adsorption of BPA in the presence of CTAB was affected less by the pore blockage effects compared with that of EE2 because of the smaller molecular size and deformable structure of BPA. These results are of great importance for pre- dicting EDC environment behavior on GMs in the real aquatic environment.

\section{Data Availability}

All data generated or analyzed during this study are included in this published article and its supplementary information files.

\section{Conflicts of Interest}

The authors declared no potential conflicts of interest with respect to the research, authorship, and/or publication of this article.

\section{Acknowledgments}

This work was supported by the National Natural Science Foundation of China (Grant 21707101), the Opening Foundation of the Ministry of Education Key Laboratory of Pollution Processes and Environmental Criteria (Grant 2017-06), and the Innovation Team Training Plan of the Tianjin Education Committee (TD13-5073).

\section{Supplementary Materials}

Procedures used to determine relative hydrophobicity of GMs, procedures of negligible depletion-solid-phase microextraction, and the adsorption isotherm models of EDC are shown in the Supplemental Material. Table S1: shows the summary of adsorbate properties. Table S2: shows critical micelle concentration $(\mathrm{CMC})$ and molecular structures of surfactants. Table S3: shows the zeta potential of GO and RGOs in the absence and presence of surfactants. Figure S1: shows fiber calibrations of EE2 and BPA in the absence and presence of CTAB. Figure S2: shows the adsorption isotherms of EE2 and BPA to GMs further normalized by relative hydrophobicity index $(\mathrm{a}, \mathrm{b})$ and $I_{\mathrm{D}} / I_{\mathrm{G}}(\mathrm{c}, \mathrm{d})$ of GMs, respectively. Figure $\mathrm{S} 3$ : shows comparison of the effects of CTAB on solubility of EE2 and BPA. Figure S4: shows the adsorbed amount $(q)$ and adsorption affinities $\left(\log K_{\mathrm{d}}\right)$ of CTAB on GMs. Supplemental material for this article is available online. (Supplementary Materials)

\section{References}

[1] E. Archer, B. Petrie, B. Kasprzyk-Hordern, and G. M. Wolfaardt, "The fate of pharmaceuticals and personal care products (PPCPs), endocrine disrupting contaminants (EDCs), metabolites and illicit drugs in a WWTW and environmental waters," Chemosphere, vol. 174, pp. 437-446, 2017.

[2] A. Z. Aris, Z. A. M. Hir, and M. R. Razak, "Metal-organic frameworks (MOFs) for the adsorptive removal of selected endocrine disrupting compounds (EDCs) from aqueous solution: a review," Applied Materials Today, vol. 21, article 100796, 2020.

[3] R. Wang, E. Biles, Y. Li et al., "In situ catchment scale sampling of emerging contaminants using diffusive gradients in thin films (DGT) and traditional grab sampling: a case study of 
the River Thames, UK," Environmental Science \& Technology, vol. 54, no. 18, pp. 11155-11164, 2020.

[4] S. Yang, W. Yu, L. Yang et al., "Occurrence and fate of steroid estrogens in a Chinese typical concentrated dairy farm and slurry irrigated soil," Journal of Agricultural \& Food Chemistry, vol. 69, no. 1, pp. 67-77, 2021.

[5] W. Yu, B. Du, L. Yang et al., "Occurrence, sorption, and transformation of free and conjugated natural steroid estrogens in the environment," Environmental Science \& Pollution Research, vol. 26, no. 10, pp. 9443-9468, 2019.

[6] R. Sacdal, J. Madriaga, and M. P. Espino, "Overview of the analysis, occurrence and ecological effects of hormones in lake waters in Asia," Environmental Research, vol. 182, article 109091, 2020.

[7] X. Zhou, Y. Li, H. Li, Z. Yang, and C. Zuo, "Responses in the crucian carp (Carassius auratus) exposed to environmentally relevant concentration of $17 \alpha$-ethinylestradiol based on metabolomics," Ecotoxicology \& Environmental Safety, vol. 183, article 109501, 2019.

[8] U. R. Farooqui, A. L. Ahmad, and N. A. Hamid, "Graphene oxide: a promising membrane material for fuel cells," Renewable \& Sustainable Energy Reviews, vol. 82, pp. 714-733, 2018.

[9] K. Fu, Y. Yao, J. Dai, and L. Hu, "Progress in 3D printing of carbon materials for energy-related applications," Advanced Materials, vol. 29, no. 9, article 1603486, 2017.

[10] C. Huang, C. Li, and G. Shi, "Graphene based catalysts," Energy \& Environmental Science, vol. 5, no. 10, pp. 88488868, 2012.

[11] A. Khan, J. Wang, J. Li et al., "The role of graphene oxide and graphene oxide-based nanomaterials in the removal of pharmaceuticals from aqueous media: a review," Environmental Science \& Pollution Research, vol. 24, no. 9, pp. 7938-7958, 2017.

[12] X. Zhou and F. Liang, "Application of graphene/graphene oxide in biomedicine and biotechnology," Current Medicinal Chemistry, vol. 21, no. 7, pp. 855-869, 2014.

[13] L. Jiang, Y. Liu, S. Liu et al., "Adsorption of estrogen contaminants by graphene nanomaterials under natural organic matter preloading: comparison to carbon nanotube, biochar, and activated carbon," Environmental Science \& Technology, vol. 51, no. 11, pp. 6352-6359, 2017.

[14] L. Jiang, Y. Liu, G. Zeng et al., "Removal of $17 \beta$-estradiol by few-layered graphene oxide nanosheets from aqueous solutions: external influence and adsorption mechanism," Chemical Engineering Journal, vol. 284, pp. 93-102, 2016.

[15] Z. Jin, X. Wang, Y. Sun, Y. Ai, and X. Wang, "Adsorption of 4n-nonylphenol and bisphenol-A on magnetic reduced graphene oxides: a combined experimental and theoretical studies," Environmental Science \& Technology, vol. 49, no. 15, pp. 9168-9175, 2015.

[16] S. Kim, C. M. Park, M. Jang et al., “Aqueous removal of inorganic and organic contaminants by graphene-based nanoadsorbents: a review," Chemosphere, vol. 212, pp. 1104-1124, 2018.

[17] P. Borthakur, P. K. Boruah, M. R. Das, N. Kulik, and B. Minofar, "Adsorption of $17 \alpha$-ethynyl estradiol and $\beta$-estradiol on graphene oxide surface: an experimental and computational study," Journal of Molecular Liquids, vol. 269, pp. 160168, 2018.

[18] W. Sun, M. Li, W. Zhang, J. Wei, B. Chen, and C. Wang, "Sediments inhibit adsorption of $17 \beta$-estradiol and $17 \alpha$-ethinyles- tradiol to carbon nanotubes and graphene oxide," Environmental Science: Nano, vol. 4, no. 9, pp. 1900-1910, 2017.

[19] W. Sun, C. Wang, W. Pan, S. Li, and B. Chen, "Effects of natural minerals on the adsorption of $17 \beta$-estradiol and bisphenol A on graphene oxide and reduced graphene oxide," Environmental Science: Nano, vol. 4, no. 6, pp. 1377-1388, 2017.

[20] D. Wei, C. Zhao, A. Khan et al., "Sorption mechanism and dynamic behavior of graphene oxide as an effective adsorbent for the removal of chlorophenol based environmental-hormones: a DFT and MD simulation study," Chemical Engineering Journal, vol. 375, article 121964, 2019.

[21] Q. Gao, F. Wu, J. Hu et al., "Chemical composition-dependent removal of cationic surfactants by carbon nanotubes," Science of the Total Environment, vol. 716, article 137017, 2020.

[22] A. Kierkegaard, C. Chen, J. M. Armitage, J. A. Arnot, S. Droge, and M. S. McLachlan, "Tissue distribution of several series of cationic surfactants in rainbow trout (Oncorhynchus mykiss) following exposure via water," Environmental Science \& Technology, vol. 54, no. 7, pp. 4190-4199, 2020.

[23] S. Koner, A. Pal, and A. Adak, "Utilization of silica gel waste for adsorption of cationic surfactant and adsolubilization of organics from textile wastewater: a case study," Desalination, vol. 276, no. 1-3, pp. 142-147, 2011.

[24] M. Lavorgna, C. Russo, B. D'Abrosca, A. Parrella, and M. Isidori, "Toxicity and genotoxicity of the quaternary ammonium compound benzalkonium chloride (BAC) using Daphnia magna and Ceriodaphnia dubia as model systems," Environmental Pollution, vol. 210, pp. 34-39, 2016.

[25] B. Mondal, A. Adak, and P. Datta, "Effect of operating conditions and interfering substances on photochemical degradation of a cationic surfactant," Environmental Technology, vol. 39, no. 21, pp. 2771-2780, 2018.

[26] A. A. Siyal, M. R. Shamsuddin, and A. Low, "Fly ash based geopolymer for the adsorption of cationic and nonionic surfactants from aqueous solution-a feasibility study," Materials Letters, vol. 283, article 128758, 2021.

[27] F. Zhang, S. Li, Q. Zhang et al., "Adsorption of different types of surfactants on graphene oxide," Journal of Molecular Liquids, vol. 276, pp. 338-346, 2019.

[28] K. Yang, Q. Jing, W. Wu, L. Zhu, and B. Xing, “Adsorption and conformation of a cationic surfactant on single-walled carbon nanotubes and their influence on naphthalene sorption," Environmental Science \& Technology, vol. 44, no. 2, pp. 681-687, 2010.

[29] B. Pan, D. Lin, H. Mashayekhi, and B. Xing, "Adsorption and hysteresis of bisphenol A and $17 \alpha$-ethinyl estradiol on carbon nanomaterials," Environmental Science \& Technology, vol. 42, no. 15 , pp. 5480-5485, 2008.

[30] J. Zhao, Z. Wang, Q. Zhao, and B. Xing, “Adsorption of phenanthrene on multilayer graphene as affected by surfactant and exfoliation," Environmental Science \& Technology, vol. 48, no. 1, pp. 331-339, 2014.

[31] P. Oleszczuk and B. Xing, "Influence of anionic, cationic and nonionic surfactants on adsorption and desorption of oxytetracycline by ultrasonically treated and non-treated multiwalled carbon nanotubes," Chemosphere, vol. 85, no. 8, pp. 1312-1317, 2011.

[32] F. Wang, Z. Jia, W. Su, Y. Shang, and Z. L. Wang, "Adsorption of phenanthrene and 1-naphthol to graphene oxide and (L)ascorbic-acid-reduced graphene oxide: effects of $\mathrm{pH}$ and 
surfactants," Environmental Science \& Pollution Research, vol. 26, no. 11, pp. 11062-11073, 2019.

[33] S. L. Walker, J. E. Hill, J. A. Redman, and M. Elimelech, "Influence of growth phase on adhesion kinetics of Escherichia coli D21g," Applied \& Environmental Microbiology, vol. 71, no. 6, pp. 3093-3099, 2005.

[34] F. Wang, J. J.-H. Haftka, T. L. Sinnige, J. L. M. Hermens, and W. Chen, "Adsorption of polar, nonpolar, and substituted aromatics to colloidal graphene oxide nanoparticles," Environmental Pollution, vol. 186, pp. 226-233, 2014.

[35] Y. H. Ding, P. Zhang, Q. Zhuo, H. M. Ren, Z. M. Yang, and Y. Jiang, "A green approach to the synthesis of reduced graphene oxide nanosheets under UV irradiation," Nanotechnology, vol. 22, no. 21, article 215601, 2011.

[36] H. Jung, S. J. Yang, T. Kim, J. H. Kang, and C. R. Park, “Ultrafast room-temperature reduction of graphene oxide to graphene with excellent dispersibility by lithium naphthalenide," Carbon, vol. 63, pp. 165-174, 2013.

[37] C. Chen, Y.-C. Chen, Y.-T. Hong, T. W. Lee, and J. F. Huang, "Facile fabrication of ascorbic acid reduced graphene oxidemodified electrodes toward electroanalytical determination of sulfamethoxazole in aqueous environments," Chemical Engineering Journal, vol. 352, pp. 188-197, 2018.

[38] S. Stankovich, D. A. Dikin, R. D. Piner et al., "Synthesis of graphene-based nanosheets via chemical reduction of exfoliated graphite oxide," Carbon, vol. 45, no. 7, pp. 1558-1565, 2007.

[39] J. I. Paredes, A. Martínez-Alonso, T. Yamazaki, K. Matsuoka, J. M. D. Tascón, and T. Kyotani, "Structural investigation of zeolite-templated, ordered microporous carbon by scanning tunneling microscopy and Raman spectroscopy," Langmuir, vol. 21, no. 19, pp. 8817-8823, 2005.

[40] L. Joseph, J. Heo, Y.-G. Park, J. R. V. Flora, and Y. Yoon, "Adsorption of bisphenol A and 17 $\alpha$-ethinyl estradiol on single walled carbon nanotubes from seawater and brackish water," Desalination, vol. 281, pp. 68-74, 2011.

[41] J. Xu, L. Wang, and Y. Zhu, "Decontamination of bisphenol A from aqueous solution by graphene adsorption," Langmuir, vol. 28 , no. 22, pp. 8418-8425, 2012.

[42] F. Liu, J. Zhao, S. Wang, P. du, and B. Xing, "Effects of solution chemistry on adsorption of selected pharmaceuticals and personal care products (PPCPs) by graphenes and carbon nanotubes," Environmental Science \& Technology, vol. 48, no. 22, pp. 13197-13206, 2014.

[43] J. Zhao, Z. Wang, H. Mashayekhi, P. Mayer, B. Chefetz, and B. Xing, "Pulmonary surfactant suppressed phenanthrene adsorption on carbon nanotubes through solubilization and competition as examined by passive dosing technique," Environmental Science \& Technology, vol. 46, no. 10, pp. 53695377, 2012.

[44] F. Wang, F. Wang, D. Zhu, and W. Chen, "Effects of sulfide reduction on adsorption affinities of colloidal graphene oxide nanoparticles for phenanthrene and 1-naphthol," Environmental Pollution, vol. 196, pp. 371-378, 2015. 